\title{
Reduced Neutralization of SARS-CoV-2 Omicron Variant by Vaccine Sera and Monoclonal Antibodies
}

\author{
Alexander Wilhelm ${ }^{1 *}$, Marek Widera ${ }^{1 *}$, Katharina Grikscheit ${ }^{1}$, Tuna Toptan ${ }^{1}$, Barbara Schenk ${ }^{1}$, Christiane Pallas $^{1}$, \\ Melinda Metzler ${ }^{1}$, Niko Kohmer ${ }^{1}$, Sebastian Hoehl ${ }^{1}$, Fabian A. Helfritz ${ }^{2}$, Timo Wolf $^{3}$, Udo Goetsch ${ }^{4}$, Sandra \\ Ciesek ${ }^{1,5,6 \#}$ \\ ${ }^{1}$ Institute for Medical Virology, University Hospital Frankfurt, Goethe University Frankfurt, 60596 Frankfurt am Main, \\ Germany \\ ${ }^{2}$ Bürgerhospital Frankfurt, Nibelungenallee 37-41, 60318 Frankfurt am Main, Germany \\ ${ }^{3}$ Department of Internal Medicine, Infectious Diseases, University Hospital Frankfurt, Goethe University Frankfurt, \\ 60596 Frankfurt am Main, Germany \\ ${ }^{4}$ Health Protection Authority of the City of Frankfurt am Main, 60313 Frankfurt am Main, Germany \\ ${ }^{5}$ German Center for Infection Research (DZIF), 38124 Braunschweig, Germany \\ ${ }^{6}$ Branch Translational Medicine and Pharmacology, Fraunhofer Institute for Molecular Biology and Applied Ecology \\ (IME), 60596 Frankfurt am Main, Germany \\ ${ }^{*}$ contributed equally \\ \# Corresponding author: Sandra Ciesek M.D. (sandra.ciesek@kgu.de)
}

v4, updated 2021-12-11

\begin{abstract}
:
Due to numerous mutations in the spike protein, the SARS-CoV-2 variant of concern Omicron (B.1.1.529) raises serious concerns since it may significantly limit the antibody-mediated neutralization and increase the risk of reinfections. While a rapid increase in the number of cases is being reported worldwide, until now there has been uncertainty about the efficacy of vaccinations and monoclonal antibodies. Our in vitro findings using authentic SARS-CoV-2 variants indicate that in contrast to the currently circulating Delta variant, the neutralization efficacy of vaccine-elicited sera against Omicron was severely reduced highlighting T-cell mediated immunity as essential barrier to prevent severe COVID-19. Since SARS-CoV-2 Omicron was resistant to casirivimab and imdevimab, genotyping of SARS-CoV-2 may be needed before initiating mAb treatment. Variant-specific vaccines and $\mathrm{mAb}$ agents may be required to treat COVID-19 due to Omicron and other emerging variants of concern.
\end{abstract}


The SARS-CoV-2 variant Omicron was first identified in South Africa on November 9, 2021. Due to numerous mutations in the spike protein (S), which is the antigenic target of vaccine-elicited antibodies, Omicron raises serious concerns about significantly reduced vaccine efficacy and increased risk of reinfection ${ }^{1}$. Compared to the parental variant (B.1), Omicron $S$ has 30 non-synonymous substitutions, three small deletions and an insertion (Supplementary Figure 1, Supplementary Tables 1-3). Fifteen of these mutations are in the receptor-binding domain (RBD), a major target of neutralizing antibodies (NAbs) ${ }^{2}$. Several of the $S$ mutations observed in Omicron were reported in preceding variants of concern (VoCs) like Alpha, Beta, Gamma, Delta as well as variants of interest such as Kappa, Zeta, Lambda, and Mu (Supplementary Table 3) that were associated with higher transmissibility and immune escape. So far, Beta and Mu exhibited the most severe immune evading capacities ${ }^{3,4}$. Due to the high accumulation of these mutations in Omicron S, synergistic effects are expected and it is unclear whether prior immunity protects against re-infections.

To evaluate the protective capacity, antibody-mediated neutralization efficacy against authentic SARS-CoV-2 Omicron was determined in vitro using an isolate obtained from a double mRNA-1273-vaccinated travel returnee from Zimbabwe and compared to Delta. Neutralization performed with sera from double or triple BNT162b2vaccinated individuals $(6,0.5$ or 3 months after last vaccination/booster) revealed an 11.4-, 37.0- and 24.5-fold reduction, respectively (Figure 1A). Sera from double mRNA-1273-vaccinated and additionally BNT162b2vaccinated individuals (sampled 6 or 0.5 months after last vaccination/booster) showed a 20 - and 22.7-fold reduction in the neutralization capacity (Figure 1B). Poor neutralization against Delta and no efficacy against Omicron were observed using sera from heterologous ChAdOx1/BNT162b2-vaccinated individuals (Figure 1C). Additionally, the group receiving a third BNT162b2-vaccination showed a significant increase of NAb titers but a 27.1-fold reduction in neutralization against Omicron (Figure 1C). Neutralization of Omicron was 32.8-fold reduced using sera from double BNT162b2-vaccinated and previously SARS-CoV-2-infected individuals (Figure 1A).

The currently used monoclonal antibodies $(\mathrm{mAb})$ imdevimab and casirivimab efficiently prevented Delta infection, however, most likely in consequence of amino acid substitutions ${ }^{5}$ failed to neutralize Omicron (Figure 1D). In contrast to the currently circulating Delta variant, neutralization efficacy of vaccine-elicited sera against Omicron was severely reduced highlighting T-cell mediated immunity as essential barrier to prevent severe COVID-19. Since Omicron was resistant to casirivimab and imdevimab SARS-CoV-2 genotyping may be needed before initiating $m A b$ treatment. Booster vaccinations, variant-specific vaccines and mAb agents may be required to treat COVID-19 with emerging variants of concern. 
A

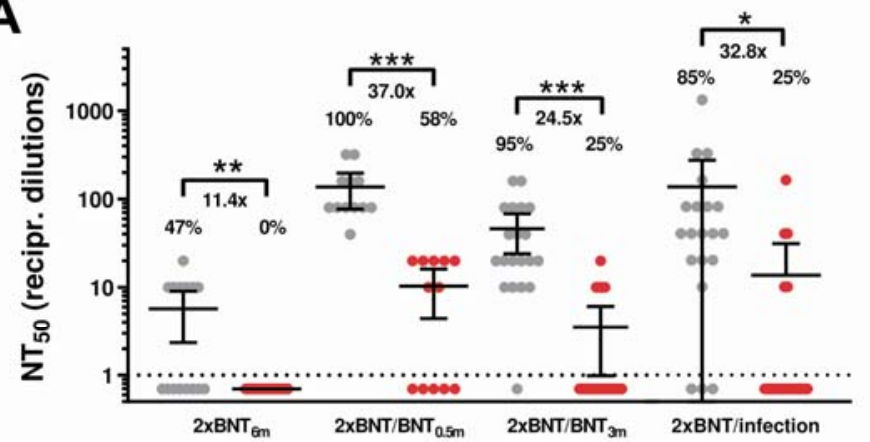

B

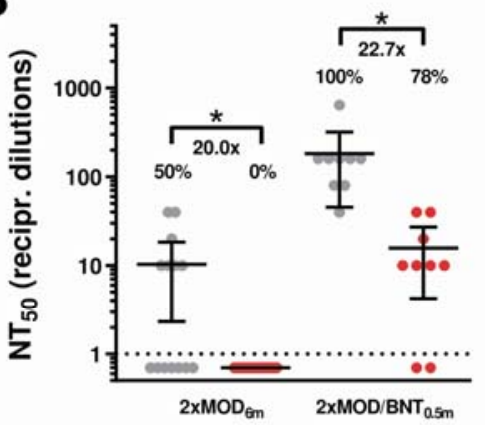

C

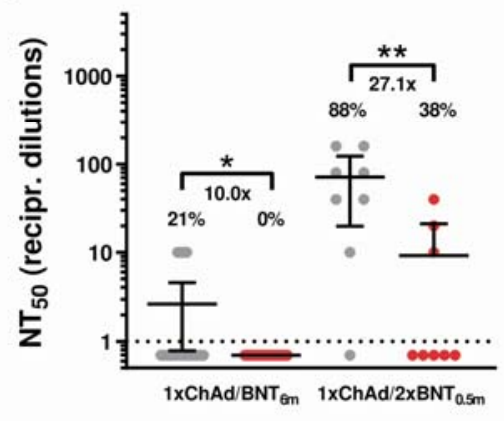

D

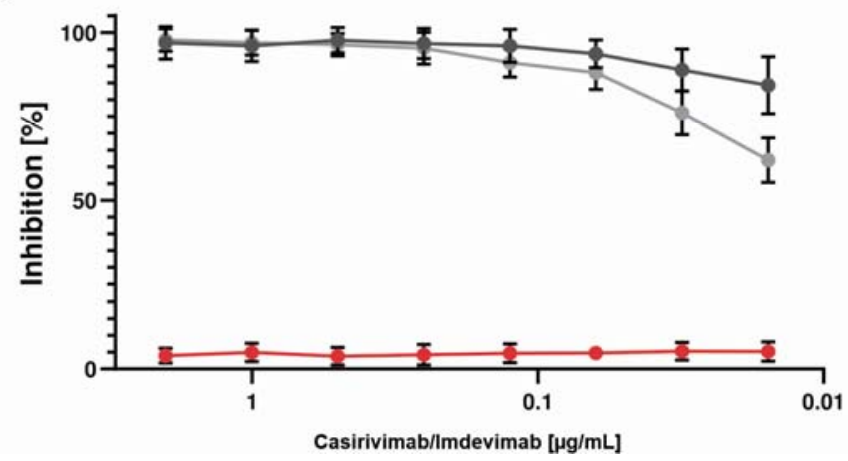

Figure 1 - Antibody-mediated neutralization efficacy against authentic SARS-CoV-2 variants Delta and Omicron. Values represent reciprocal dilutions of SARS-CoV-2 variants Delta (grey) and Omicron (red) microneutralization titers resulting in $50 \%$ virus neutralization $\left(\mathrm{NT}_{50}\right)$. A) Neutralization assays were performed using serum samples obtained from vaccinated individuals receiving the indicated vaccine schemes (sampling time after last vaccination/booster indicated in subscript): double BNT162b2-vaccinated (2xBNT $6 \mathrm{~m})$, sera from triple BNT162b2-vaccinated individuals (2xBNT/BNT ${ }_{0.5 \mathrm{~m}}$ and $2 \times \mathrm{BNT} \mathrm{BNT}_{3 \mathrm{~m}}$ ) and double BNT162b2-vaccinated and SARS-CoV-2 infected individuals (2xBNT/infection). B) Neutralization assays with sera from double mRNA-1273vaccinated $\left(2 \mathrm{xMOD}_{6 \mathrm{~m}}\right)$ and additionally BNT162b2-boosted $\left(2 \times M O D / B N T_{0.5 \mathrm{~m}}\right)$. C) Neutralization titers for sera from heterologous ChAdOx1 and BNT162b2-vaccinated (1xChAd/1xBNT $\left.{ }_{6 m}\right)$ and BNT162b2 boosted (1xChAd/2xBNT $\left.0.5 \mathrm{~m}\right)$ individuals. The $\mathrm{x}$-fold reduction was determined using the difference between $\mathrm{NT}_{50}$ values for Delta and Omicron. Only Delta neutralizing samples were considered and negative titers were handled as 1 for fold-change calculation. The percentages indicate the relative number of sera that yielded a measurable titer. Information regarding the sera donors (sex, age, antibody titers test and time after vaccination) are summarized in Supplementary Table 4. D) Neutralization efficacy of monoclonal antibodies imdevimab and casirivimab against SARS-CoV-2 Omicron (red), parental strain B (dark grey), and Delta (grey). The indicated concentrations of mAbs casirivimab and imdevimab were applied in a 1:1 ratio. Mean values of two technical replicates per sample are depicted with $95 \%$ confidence intervals and SD. All experiments were verified using a second SARS-CoV-2 Omicron strain (EPI_ISL_6959868; Supplementary Figure 1). Statistical significance compared to Delta was calculated by two-tailed, paired student's t-tests. Asterisks indicate $p$-values as ${ }^{*}(p<0.05),{ }^{* *}(p<0.01)$, and ${ }^{* * *}(p<0.001)$.

\section{Materials and Methods}

\section{Ethics statement}

The study was conducted according to the guidelines of the Declaration of Helsinki, and approved by the Institutional Review Board of the Ethics Committee of the Faculty of Medicine at Goethe University Frankfurt (2021-201, 20-864 and 250719).

\section{Human sera}

Peripheral blood was collected from vaccinated individuals as indicated in Supplementary Table 4. All sera were prepared by centrifugation $2000 \mathrm{xg}$ for $10 \mathrm{~min}$, inactivated at $56^{\circ} \mathrm{C}$ for $30 \mathrm{~min}$, and stored at $-20^{\circ} \mathrm{C}$ until 
use.

\section{Virus identification and Sequencing}

SARS-CoV-2 isolates were obtained from nasopharyngeal swabs of travel returnees from South Africa as screened by the Public Health Department of the City of Frankfurt am Main, Germany. Swab material was suspended in $1.5 \mathrm{~mL}$ phosphate-buffered saline (PBS) and split for RNA-Isolation and viral outgrowth assay. RNA was isolated using the QIAamp Viral RNA Mini Kit (QIAGEN, Hilden, Germany) according to manufacturer's instructions. RNA was subjected to variant specific RT-qPCR genotyping and Oxford Nanopore sequencing.

\section{Library preparation, sequencing and bioinformatics analysis}

RNA samples extracted from swabs were used for library preparation according to NEBNext ARTIC Standard Protocol (New England Biolabs Ipswich, Massachusetts, USA) (dx.doi.org/10.17504/protocols.io.budxns7n) using the Artic nCoV-2019 V4 primers (IDT, Coralville, lowa, USA). Libraries were generated using ligation sequencing kit SQK-LSK109, native barcoding expansion kit EXP-NBD104 and FLO-MIN106D R9.4.1 flow cell according to the standard protocol (Oxford Nanopore Technologies, UK) and sequenced on MinION MK1c (Oxford Nanopore Technologies, UK) for $8 \mathrm{~h}$ with basecalling and demultiplexing options enabled. The obtained FASTQ files were filtered and analyzed using ARTIC pipeline (https://artic.network/ncov-2019/ncov2019bioinformatics-sop.html).

See Supplementary Figure 1 for schematic representation of the SARS-CoV-2 genome indicating spike positions. Sequences are available on GISAID and GenBank under the following accession numbers:

SARS-CoV-2 B.1.1.529 FFM-SIM0550/2021 (EPI_ISL_6959871; GenBank ID: OL800702),

SARS-CoV-2 B.1.1.529 FFM-ZAF0396/2021 (EPI_ISL_6959868; GenBank ID: OL800703),

SARS-CoV-2 B.1.617.2 FFM-IND8424/2021 (GenBank ID: MZ315141).

\section{Cell culture and Virus Propagation}

A549-AT cells ${ }^{6}$ stably expressing ACE2 and TMPRSS2 and Caco2 cells (DSMZ, Braunschweig, Germany, no: ACC 169) were maintained in Minimum Essential Medium (MEM) supplemented with $10 \%$ fetal calf serum (FCS), $4 \mathrm{mM} \mathrm{L}$-glutamine, $100 \mathrm{IU} / \mathrm{mL}$ of penicillin, and $100 \mu \mathrm{g} / \mathrm{mL}$ of streptomycin at $37^{\circ} \mathrm{C}$ and $5 \% \mathrm{CO}_{2}$. All culture reagents were purchased from Sigma (St. Louis, MO, USA).

As described previously SARS-CoV-2 isolates were propagated using Caco2 cells, which were selected for high permissiveness to SARS-CoV-2 infection by serial dilution and passaging as described previously ${ }^{7}$. Cellfree cell culture supernatant containing infectious virus was harvested after complete cytopathic effect (CPE) and aliquots were stored at $-80^{\circ} \mathrm{C}$. Titers were determined by the median tissue culture infective dose $\left(\mathrm{TCID}_{50}\right)$ method as described by Spearman ${ }^{8}$ and Kaerber $^{9}$ using Caco2 cells. All cell culture work involving infectious SARS-CoV-2 was performed under biosafety level 3 (BSL-3) conditions. Sample inactivation for further processing was performed with previously evaluated methods ${ }^{10}$.

\section{Neutralization and antiviral assays}

SARS-CoV-2 antibody concentrations were determined using the SARS-CoV-2 IgG II Quant assay and the Alinity I device (Abbott Diagnostics, Wiesbaden, Germany) with an analytical measurement range from 2.985680 binding antibody units per $\mathrm{mL}(\mathrm{BAU} / \mathrm{mL})$. All sera were initially $1: 10$ and subsequently serially $1: 2$ diluted and incubated with $4000 \mathrm{TCID}_{50} / \mathrm{mL}$ of SARS-CoV-2 Delta or Omicron. Infected cells were monitored for cytopathic effect (CPE) formation $48 \mathrm{~h}$ post inoculation. Monoclonal antibody solutions containing imdevimab and casirivimab alone or in combination in equal ratios (1:1) were serially diluted (1:2) and incubated with 4000 $\mathrm{TCID}_{50} / \mathrm{mL}$ of the indicated SARS-CoV-2 variant. After $48 \mathrm{~h}$ CPE formation was evaluated microscopically. Evaluation of monoclonal antibodies was quantified using Spark Cyto 400 multimode imaging plate reader 
medRxiv preprint doi: https://doi.org/10.1101/2021.12.07.21267432; this version posted December $13,2021$. The copyright holder for this preprint (which was not certified by peer review) is the author/funder, who has granted medRxiv a license to display the preprint in perpetuity.

It is made available under a CC-BY-ND 4.0 International license .

(Tecan) as described before ${ }^{6,11}$.

\section{References:}

1. Pulliam JRC, van Schalkwyk C, Govender N, et al. Increased risk of SARS-CoV-2 reinfection associated with emergence of the Omicron variant in South Africa. medRxiv 2021:2021.11.11.21266068. DOI: 10.1101/2021.11.11.21266068.

2. Harvey WT, Carabelli AM, Jackson B, et al. SARS-CoV-2 variants, spike mutations and immune escape. Nature reviews Microbiology 2021;19(7):409-424. DOI: 10.1038/s41579-021-00573-0.

3. Uriu K, Kimura I, Shirakawa K, et al. Neutralization of the SARS-CoV-2 Mu Variant by Convalescent and Vaccine Serum. The New England journal of medicine 2021. DOI: 10.1056/NEJMc2114706.

4. Widera M, Wilhelm A, Hoehl S, et al. Limited neutralization of authentic SARS-CoV-2 variants carrying E484K in vitro. The Journal of infectious diseases 2021. DOI: 10.1093/infdis/jiab355.

5. Barnes CO, Jette CA, Abernathy ME, et al. SARS-CoV-2 neutralizing antibody structures inform therapeutic strategies. Nature 2020;588(7839):682-687. DOI: 10.1038/s41586-020-2852-1.

6. Widera M, Wilhelm A, Toptan T, et al. Generation of a Sleeping Beauty Transposon-Based Cellular System for Rapid and Sensitive Screening for Compounds and Cellular Factors Limiting SARS-CoV-2 Replication. Frontiers in microbiology 2021;12(2034):701198. (Original Research) (In English). DOI: 10.3389/fmicb.2021.701198.

7. Widera M, Muhlemann B, Corman VM, et al. Surveillance of SARS-CoV-2 in Frankfurt am Main from October to December 2020 Reveals High Viral Diversity Including Spike Mutation N501Y in B.1.1.70 and B.1.1.7. Microorganisms 2021;9(4). DOI: 10.3390/microorganisms 9040748.

8. Spearman C. The method of right and wrong cases (constant stimuli) without Gauss's formulae. British journal of psychology 1908;2(3):227.

9. Kärber G. Beitrag zur kollektiven Behandlung pharmakologischer Reihenversuche. Naunyn-Schmiedebergs Archiv für experimentelle pathologie und pharmakologie 1931;162(4):480-483.

10. Widera M, Westhaus S, Rabenau HF, et al. Evaluation of stability and inactivation methods of SARS-CoV-2 in context of laboratory settings. Medical microbiology and immunology 2021;210(4):235-244. DOI: 10.1007/s00430-021-00716-3.

11. Wilhelm A, Toptan T, Pallas C, et al. Antibody-Mediated Neutralization of Authentic SARS-CoV-2 B.1.617 Variants Harboring L452R and T478K/E484Q. Viruses 2021;13(9). DOI: 10.3390/v13091693.

12. Elbe S, Buckland-Merrett G. Data, disease and diplomacy: GISAID's innovative contribution to global health. Glob Chall 2017;1(1):33-46. DOI: 10.1002/gch2.1018.

\section{Acknowledgements:}

This study has been performed with the support of the Goethe-Corona-Fund of the Goethe University Frankfurt (MW) and the Federal Ministry of Education and Research (COVIDready; grant 02WRS1621C (MW). We are thankful for the numerous donations to the Goethe-Corona-Fund and the support of our SARS-CoV-2 research. The authors would also like to thank all technical staff involved in data acquisition. 
medRxiv preprint doi: https://doi.org/10.1101/2021.12.07.21267432; this version posted December 13, 2021. The copyright holder for this preprint (which was not certified by peer review) is the author/funder, who has granted medRxiv a license to display the preprint in perpetuity.

It is made available under a CC-BY-ND 4.0 International license .

\section{Supplementary Material:}

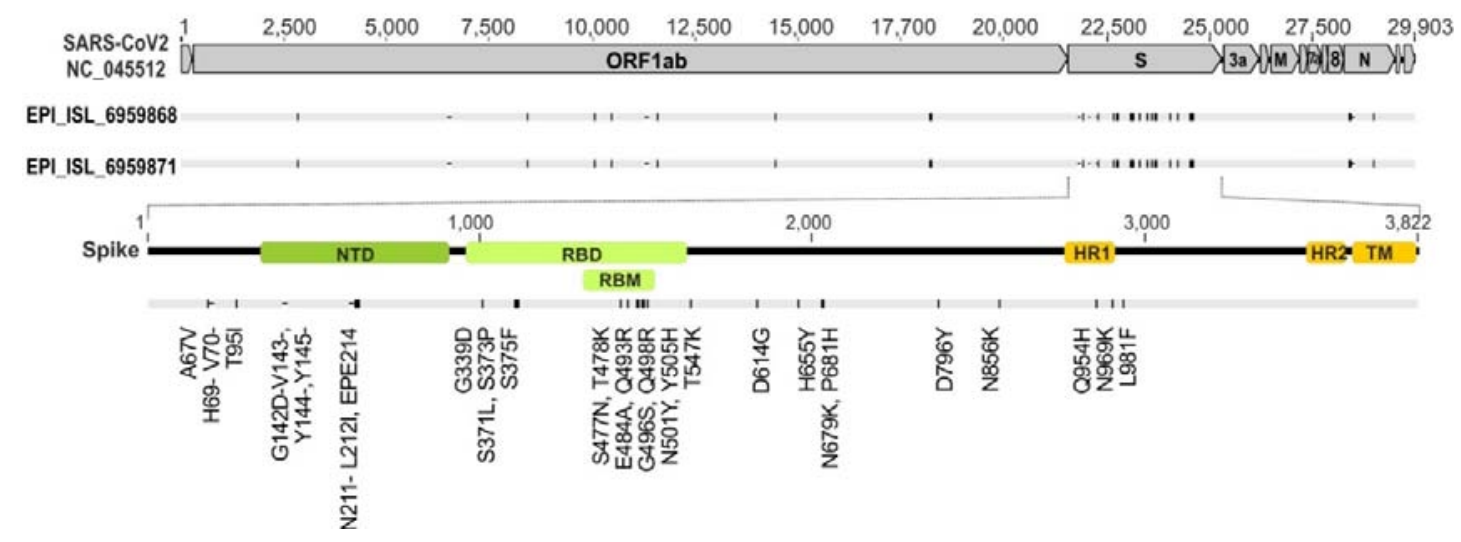

Supplementary Figure 1 - Schematic representation of the SARS-CoV-2 genome indicating spike positions. The numbers denote nucleotide positions based on the reference strain NC_045512. Primary, SARS-CoV-2 Omricon strain FFMSIM0550/2021 (GISAID: EPI ISL 6959871; GenBank ID: OL800702) was used in this study. SARS-CoV-2 Omricon strain FFMZAF0396/2021 (GISAID: EPI_ISL_6959868; GenBank ID: OL800703) was used for confirmation. NTD RBD and RBM are highlighted by green boxes. HR1, $\mathrm{HR} 2$, and TM are indicated by orange boxes. ORF based on reference sequence NC_045512 are shown as grey boxes. Nucleotide substitutions compared to the reference sequence are indicated in the lower section. For sequencing coverage see Supplementary Table 2. Dropouts and low coverage regions: Spike: (22796-22983), (23621-23885); E/M gene: (26348-27186). For affected Nanopore Primers see Supplementary Table 1.

Supplementary Table 1 - Dropouts and low coverage Regions: Spike: (22796-22983), (23621-23885) E/M gene: (26348-27186)

\begin{tabular}{|l|l|}
\hline \multicolumn{1}{|c|}{ Position in SARS-CoV-2 genome } & Affected Nanopore Primer \\
\hline 22673 & SARS-CoV-2_76_LEFT \\
\hline 22674 & SARS-CoV-2_76_LEFT \\
\hline 23040 & SARS-CoV-2_76_RIGHT \\
\hline 23048 & SARS-CoV-2_76_RIGHT \\
\hline 23055 & SARS-CoV-2_76_RIGHT \\
\hline 23948 & SARS-CoV-2_79_RIGHT \\
\hline 26270 & SARS-CoV-2_88_LEFT \\
\hline 26577 & SARS-CoV-2_89_LEFT \\
\hline 27259 & SARS-CoV-2_90_RIGHT \\
\hline
\end{tabular}


medRxiv preprint doi: https://doi.org/10.1101/2021.12.07.21267432; this version posted December 13, 2021. The copyright holder for this preprint (which was not certified by peer review) is the author/funder, who has granted medRxiv a license to display the preprint in perpetuity.

It is made available under a CC-BY-ND 4.0 International license .

Supplementary Table 2 - Sequencing coverage, identity and amino acid (aa) changes of SARS-CoV-2 Omicron samples.

\begin{tabular}{|c|c|c|c|c|}
\hline Best reference hit & \%id & $\begin{array}{l}\text { \%covera } \\
\text { ge }\end{array}$ & $\begin{array}{l}\# \Delta \\
\mathbf{s}\end{array}$ & List of aa changes \\
\hline NSP1 hCoV-19/Wuhan/WIV04/2019 & $100 \%$ & $100 \%$ & 0 & no aa changes \\
\hline NSP2 hCoV-19/Wuhan/WIV04/2019 & $100 \%$ & $100 \%$ & 0 & no aa changes \\
\hline NSP3 hCoV-19/Wuhan/WIV04/2019 & $99.8 \%$ & $99.9 \%$ & 4 & K38R, S1265del, L1266I, A1892T \\
\hline NSP4 hCoV-19/Wuhan/WIV04/2019 & $99.8 \%$ & $100 \%$ & 1 & T492I \\
\hline NSP5 hCoV-19/Wuhan/WIV04/2019 & $99.7 \%$ & $100 \%$ & 1 & $\mathrm{P} 132 \mathrm{H}$ \\
\hline NSP6 hCoV-19/Wuhan/WIV04/2019 & $99.7 \%$ & $99.0 \%$ & 4 & L105del, S106del, G107del, I189V \\
\hline NSP7 hCoV-19/Wuhan/WIV04/2019 & $100 \%$ & $100 \%$ & 0 & no aa changes \\
\hline NSP8 hCoV-19/Wuhan/WIV04/2019 & $100 \%$ & $100 \%$ & 0 & no aa changes \\
\hline NSP9 hCoV-19/Wuhan/WIV04/2019 & $100 \%$ & $100 \%$ & 0 & no aa changes \\
\hline NSP10 hCoV-19/Wuhan/WIV04/2019 & $100 \%$ & $100 \%$ & 0 & no aa changes \\
\hline NSP11 hCoV-19/Wuhan/WIV04/2019 & $100 \%$ & $100 \%$ & 0 & no aa changes \\
\hline NSP12 hCoV-19/Wuhan/WIV04/2019 & $99.9 \%$ & $99.0 \%$ & 1 & P323L \\
\hline NSP13 hCoV-19/Wuhan/WIV04/2019 & $100 \%$ & $100 \%$ & 0 & no aa changes \\
\hline NSP14 hCoV-19/Wuhan/WIV04/2019 & $99.8 \%$ & $100 \%$ & 1 & $142 \mathrm{~V}$ \\
\hline NSP15 hCoV-19/Wuhan/WIV04/2019 & $100 \%$ & $100 \%$ & 0 & no aa changes \\
\hline NSP16 hCoV-19/Wuhan/WIV04/2019 & $100 \%$ & $100 \%$ & 0 & no aa changes \\
\hline NS3 hCoV-19/Wuhan/WIV04/2019 & $100 \%$ & $100 \%$ & 0 & no aa changes \\
\hline E hCoV-19/Wuhan/WIV04/2019 & $87.1 \%$ & $41.3 \%$ & 4 & T9I, A22X, F23X, F26X \\
\hline (no ref hits for $M$ ) & $0 \%$ & $0 \%$ & 0 & no coverage \\
\hline NS6 hCoV-19/Wuhan/WIV04/2019 & $100 \%$ & $100 \%$ & 0 & no aa changes \\
\hline NS7a hCoV-19/Wuhan/WIV04/2019 & $100 \%$ & $100 \%$ & 0 & no aa changes \\
\hline NS7b hCoV-19/Wuhan/WIV04/2019 & $100 \%$ & $100 \%$ & 0 & no aa changes \\
\hline NS8 hCoV-19/Wuhan/WIV04/2019 & $100 \%$ & $100 \%$ & 0 & no aa changes \\
\hline N hCoV-19/Wuhan/WIV04/2019 & $99.3 \%$ & $99.3 \%$ & 6 & $\begin{array}{l}\text { P13L, E31del, R32del, S33del, R203K, G2 } \\
\text { 04R }\end{array}$ \\
\hline
\end{tabular}


Supplementary Table 3. Mutations in the spike proteins of SARS-CoV-2 variants of concern. Compared to the parental SARS-CoV-2 isolate B.1, Omicron gains additional substitutions, insertions and deletions. Amino acid substitutions already found in other variants are highlighted in red. Altered positions but with different distinct substitutions are indicated in blue.

Parental: B.1 D614G

Alpha: $\quad$ B.1.1.7 HV69-70del, Y144del, N501Y, A570D, D614G, P681H, T716I, S982A, D1118H

Beta: $\quad$ B.1.351 L18F, D80A, D215G, R246I, K417N, E484K, N501Y, D614G, A701V

Gamma: P.1 L18F, T20N, P26S, D138Y, R190S, K417T, E484K, N501Y, D614G, H655Y, T10277I

Delta: $\quad$ B.1.617.2 T19R, T95I, G142D, E156-, F157-, R158G, L452R, T478K, D614G, P681R, D950N

Omicron: $\quad$ B.1.1.529 A67V, HV69-70del, T95I, del142-144, Y145D, del211, L212I, ins214EPE, G339D, S371L, S373P, S375F, K417N, N440K, 446S, S477N, T478K, E484A, Q493R, G496S, Q498R, N501Y, Y505H, T547K, D614G, H655Y, N679K, P681H, N764K, D796Y, N856K, Q954H, N969K, L981F

GISAID ${ }^{12}$ Lineage Comparison: Alaa Abdel Latif, Julia L. Mullen, Manar Alkuzweny, Ginger Tsueng, Marco Cano, Emily Haag, Jerry Zhou, Mark Zeller, Emory Hufbauer, Nate Matteson, Chunlei Wu, Kristian G. Andersen, Andrew I. Su, Karthik Gangavarapu, Laura D. Hughes, and the Center for Viral Systems Biology. outbreak.info (available at https://outbreak.info/compare-lineages?pango=B.1.617.2\&pango=P.1\&pango=B.1.351\&pango=B.1.1.7\&pango=B.1\&pango=Omicron \&gene=S\& threshold=94\&sub=true\&dark=false). Accessed 7 December 2021. 
Supplementary Table 4. Patient characteristics and overview of sera used in this study.

\begin{tabular}{|c|c|c|c|c|c|c|c|c|c|}
\hline Group & $\begin{array}{l}\text { Immunization } \\
\text { scheme }\end{array}$ & $\begin{array}{l}\text { Group } \\
\text { size } \\
\mathbf{n}\end{array}$ & $\begin{array}{c}\text { Age } \\
\text { Median } \\
\text { (range) }\end{array}$ & $\begin{array}{l}\text { Sex } \\
(f / m)\end{array}$ & $\begin{array}{c}\text { BAU/mL } \\
\text { Median } \\
\text { (IQR) }\end{array}$ & $\begin{array}{c}\text { BAU/mL } \\
\text { Mean (range) }\end{array}$ & $\begin{array}{l}\text { months } \\
\text { after last } \\
\text { vaccination }\end{array}$ & $\begin{array}{l}\text { months } \\
\text { after SARS- } \\
\text { CoV-2 } \\
\text { infection }\end{array}$ & $\begin{array}{l}\text { NT (Delta } \\
\text { /Omicron) } \\
\text { Median } \\
\text { (range) }\end{array}$ \\
\hline $\begin{array}{l}\text { 2xBNT/ } \\
\text { BNT } 0.5 \mathrm{~m}\end{array}$ & $\begin{array}{c}\text { 2x BNT162b + } \\
\text { 1x BNT162b }\end{array}$ & 12 & $\begin{array}{c}38 \\
(28-59) \\
\end{array}$ & $10 / 2$ & $\begin{array}{c}2143 \\
(1355-4033) \\
\end{array}$ & $\begin{array}{c}2729 \\
(825.3-6250) \\
\end{array}$ & 0.5 & 0 & $\begin{array}{c}80(40-320) / \\
10(0-20)\end{array}$ \\
\hline $\begin{array}{l}\text { 2xBNT// } \\
\text { BNT }_{3 \mathrm{~m}}\end{array}$ & $\begin{array}{c}\text { 2x BNT162b + } \\
\text { 1x BNT162b }\end{array}$ & 20 & $\begin{array}{c}43 \\
(26-63) \\
\end{array}$ & $12 / 8$ & $\begin{array}{c}1354 \\
(690.4-2178) \\
\end{array}$ & $\begin{array}{c}1838 \\
(167.1-7377) \\
\end{array}$ & $3(+/-0.8)$ & 0 & $\begin{array}{c}20(0-260) / \\
0(0-20)\end{array}$ \\
\hline $2 \mathrm{XMOD}_{6 \mathrm{~m}}$ & 2x mRNA-1273 & 14 & $\begin{array}{c}28 \\
(23-50)\end{array}$ & $7 / 7$ & $\begin{array}{c}355 \\
(174-691.1)\end{array}$ & $\begin{array}{c}588.8 \\
(90.6-2364)\end{array}$ & 6 & 0 & $\begin{array}{c}5(0-40) / \\
0(0-0)\end{array}$ \\
\hline $\begin{array}{l}\text { 2xMOD/ } \\
\text { BNT }_{0.5 \mathrm{~m}}\end{array}$ & $\begin{array}{c}\text { 2x mRNA-1273 } \\
\text { 1x BNT162b }\end{array}$ & 9 & $\begin{array}{c}29 \\
(23-50) \\
\end{array}$ & $4 / 5$ & $\begin{array}{c}2600 \\
(1879-4089) \\
\end{array}$ & $\begin{array}{c}3155 \\
(1422-6540) \\
\end{array}$ & 0.5 & 0 & $\begin{array}{c}160(40-640) / \\
10(0-40)\end{array}$ \\
\hline $\begin{array}{l}\text { 1x ChAd / } \\
\text { 1xBNT6m }\end{array}$ & $\begin{array}{l}\text { 1x ChAdOx1 } \\
\text { 1x BNT162b }\end{array}$ & 19 & $\begin{array}{c}43 \\
(20-59)\end{array}$ & $14 / 5$ & $\begin{array}{c}162.1 \\
(95.5-236.5)\end{array}$ & $\begin{array}{c}161.9 \\
(48.6-276.4)\end{array}$ & 6 & 0 & $\begin{array}{c}0(0-10) / \\
0(0-0)\end{array}$ \\
\hline $\begin{array}{l}\text { 1x ChAd / } \\
2 \times B N T_{0.5 m}\end{array}$ & $\begin{array}{l}\text { 1x ChAdOx1 } \\
\text { 2x BNT162b }\end{array}$ & 8 & $\begin{array}{c}48 \\
(30-59)\end{array}$ & $6 / 2$ & $\begin{array}{c}1544 \\
(523.9-2931)\end{array}$ & $\begin{array}{c}1728 \\
(186.8-3676)\end{array}$ & 0.5 & 0 & $\begin{array}{c}60(0-160) / \\
0(0-40)\end{array}$ \\
\hline $\begin{array}{l}\text { 2xBNT / } \\
\text { infection }\end{array}$ & $\begin{aligned} & 2 x \text { BNT162b } \\
+ & \text { SARS-CoV-2 }\end{aligned}$ & 20 & $\begin{array}{c}87.5 \\
(68-93)\end{array}$ & $17 / 3$ & $\begin{array}{c}1065 \\
(548.7-4407) \\
\end{array}$ & $\begin{array}{c}2616 \\
(58.7-11400)\end{array}$ & $6-7$ & $0.7-7.6$ & $\begin{array}{c}40(0->1380) / \\
0(0-160)\end{array}$ \\
\hline
\end{tabular}

